\title{
Connection between the Linoleic Acid and Psychopathology: A Symmetry-Breaking Phenomenon in the Brain?
}

\author{
Massimo Cocchi ${ }^{1,2 *}$, Chiara Minuto ${ }^{1,2}$, Lucio Tonello ${ }^{1}$, Jack A. Tuszynski, \\ ${ }^{1}$ “Paolo Sotgiu” Institute for Research in Quantitative \& Quantum Psychiatry \& Cardiology, LUdeS, Lugano, \\ Switzerland \\ ${ }^{2}$ Department of Veterinary Medical Sciences, University of Bologna, Bologna, Italy \\ ${ }^{3}$ Department of Oncology, University of Alberta, Edmonton, Canada \\ ${ }^{4}$ Department of Physics, University of Alberta, Edmonton, Canada \\ Email: *massimo.cocchi@unibo.it
}

Received 28 July 2015; accepted 30 October 2015; published 2 November 2015

Copyright (C) 2015 by authors and Scientific Research Publishing Inc.

This work is licensed under the Creative Commons Attribution International License (CC BY). http://creativecommons.org/licenses/by/4.0/

(c) (i) Open Access

\section{Abstract}

In this paper we discuss the role of the fatty acid composition of brain cells and in platelets in the emergence of psychiatric disorders such as major depression and the bipolar disorder. We argue that the linoleic acid concentration plays the role of a control parameter and there is a critical value of the linoleic acid concentration that determines a transition from healthy mental state to a pathological state. We draw an analogy with symmetry breaking in physical systems where at a bifurcation point on the phase diagram, infinitesimal perturbations to the state of the system result in a transition to a new global attractor state. This is in contrast to the situation away from the critical point where cause and effect are usually found in a linear or almost linear relationship. This observation may have major implications for both diagnostics and therapeutics of mental disorders when viewed as triggered by molecular causes.

\section{Keywords}

Psychiatric Disorders, Bipolar Disorder, Depression, Fatty Acids, Linoleic Acid, Cytoskeleton, Symmetry Breaking, Quantum Physics

\section{Introduction}

Modern neuroscience relates consciousness with the synchronized firing of groups of neurons as a result of ac-

${ }^{*}$ Corresponding author.

How to cite this paper: Cocchi, M., Minuto, C., Tonello, L., \& Tuszynski, J. A. (2015). Connection between the Linoleic Acid and Psychopathology: A Symmetry-Breaking Phenomenon in the Brain? Open Journal of Depression, 4, 41-52.

http://dx.doi.org/10.4236/ojd.2015.44005 
tion potential propagation and activation via synaptic connections between neighboring neurons (Nicholls et al., 2011). Higher cognitive functions including perception, attention, decision-making, learning, memory, and consciousness have been shown to be associated with synchronized oscillations in the firing of large neuronal groups. This phenomenon is known as neuronal synchrony (Abeles \& Corticonics, 1991), and interruptions of these specifically patterned electrical signaling oscillations are observed in various diseases of the brain such as epilepsy or Parkinson's disease. However, much less is known about psychiatric disorders and their correlation with molecular level functioning and organization of the brain or indeed with structural aberration of its elementary functional unit, the neuron.

Large-scale coherent synchronization of firing events across regions of the brain results in a cascade of cognitive processes including the formation and maintenance of memory, and perceptual stabilization. These coherent neuronal firing oscillations thus cannot be simply considered a byproduct of brain function, but rather must have a specific functional role in the organization of signaling among neurons. However, it is unclear what mechanism causes human or animal cognition to result from the synchronized firing of brain cells. Much of this activity concerns the neuron's membrane and membrane-bound structures such as ion channels. Rapid electrical signals propagating via gap junctions coupled with postsynaptic potential inhibition can create temporal gaps, allowing time for the generation of action potentials via excitatory neurons. Action potentials involve waves of ionic $(\mathrm{Na} / \mathrm{K})$ currents traversing the membranes of neurons through ion channels at speeds on the order of $1 \mathrm{~m} / \mathrm{s}$. Clearly, the membrane is the substrate through which electric ionic current events in the form of solitary waves propagate and hence its structure must have an effect on how these currents propagate. The cell membrane is selectively permeable to ions and organic molecules and controls the transport of material in and out of cells, including neurons. The basic function of the cell membrane is to protect the cell from undesirable external effects, allow the passage of indispensable nutrients and microelements, and maintain the cell's structural and functional integrity. It consists of the phospholipid bilayer containing cholesterol and a variety of fatty acids with embedded proteins, some of which form ion channels. Cell membranes are involved in a variety of cellular processes such as cell adhesion, ion conductivity and cell signalling and serve as the attachment surface for various extracellular structures.

The cytoskeleton is found underlying the cell membrane in the cytoplasm and provides scaffolding for membrane proteins to anchor to, as well as forming organelles that extend from the cell. Indeed, cytoskeletal elements such as actin filaments and microtubules interact extensively and intimately with the cell membrane and its components. It is, therefore, plausible that the measurable electrical events propagating across and along the neuronal membrane affect the structures within the neuron such as the cytoskeleton, in particular its microtubules (Brown \& Tuszynski, 1997). Microtubules have been extensively investigated in connections with various aspects of cognition including memory formation, information processing as well as neurodegenerative diseases (Woolf, Priel, \& Tuszynski, 2010a; Craddock et al., 2012; Craddock et al., 2012).

As the neuronal cytoskeletal network effectively connects synapses throughout the cell, electric perturbation in the form of an ion influx at the postsynaptic density can easily propagate throughout the cytoskeletal network responding to multiple inputs. The resulting signal, integrated from these multiple inputs, affects the neuron's response through electrical control of voltage-gated ion channels controlling neural signaling and higher cognitive function in a process of vertical integration across scales. The temporal focusing of synaptic inputs by coincidence detection, can allow for communication between neurons via synchrony. Additionally, the interaction between cytoskeletal electric signaling and electromagnetic fields generated by firing neurons would effectively make the cytoskeleton an antennae network working to enhance, and amplify the neural response to a spectrum of electromagnetic fields. Overall, this conceptual framework suggests that the electrically active neuronal cytoskeleton is integral to neuron function and cognitive processes (Craddock, Priel, \& Tuszynski, 2014) implications for higher cognitive function (Woolf, Priel, \& Tuszynski, 2010b) but it co-exists and interacts with the membrane, which is a direct conduit for electrical current ion flows. It has been suggested that a parallel channel of information storage and processing may involve electronic conductivity through a protein such as tubulin (especially protein filaments) via electron hopping as has been theorized and modeled by investigating semiconducting bands of tubulin within the regular periodic microtubule lattices (Hameroff, Nip, Porter et al., 2002; Friesen, Craddock, Karla et al., 2015). What has been strangely only rarely considered in the molecular level descriptions of cognitive processes involving the cytoskeleton is the inclusion of and interaction with the cellular membrane, a structure that is critically important to maintaining all living processes in cells of all types. In this paper we bring into focus the role of membrane in psychiatric disorders. It should be noted here that the mean- 
ing of cellular membrane is focused on its fatty acid profile. In its general sense, there exist some examples of such studies, e.g. Rasenick's studies on cellular membrane G-protein interactions with tubulin (Rasenick, Donati, Popova et al., 2004) and lipid raft interaction with MTs are related to depression and suicide thus to "cognitive processes” (Donati \& Rasenick, 2003).

In particular, in our opinion the composition of its fatty acids is critically important in the incidence of major psychiatric disorders. Fully appreciating this fact and taking it into account diagnostically could be of enormous practical significance since the current classification system of mental disorders is largely subjective. It is based on a phenomenological usage of questionnaires (the latest being DSM-5) that are periodically updated but conceptually unchanged for several decades. This manual, which creates a common language for clinicians involved in the diagnosis of mental disorders, includes concise and specific criteria intended to facilitate an assessment of symptom presentations in a variety of clinical settings such as inpatient, outpatient, partial hospital, consultation-liaison, clinical, private practice, and primary care. Current classification systems do not satisfactorily explain phenomena and entities of mental illness, nor do they reliably predict them. The classification systems we currently use are mainly based on consensus views but not primarily on empirical findings. Moreover, genetic findings may be vulnerable to misclassification. Unsurprisingly, clinicians have great difficulties to predict catastrophic events such as suicides because they can only look at such retrospectively. We propose here to develop cellular/molecular-based models in order to create a more adequate explanatory framework for psychopathological phenomenology. Ideally, we wish to enlist quantum-formal actuarial tools for rigorous prospective estimation of the impact of random and potentially predictable events on the evolution of illness states and catastrophic events. (i.e. to find a balance between random and predictable factors that determine a particular event or state which come actually in effect from the whole spectrum of possible events or states). The first step in this direction is to examine how the structure and composition of the membrane may be correlated with the incidence of various psychiatric disorders.

\section{Fatty Acids and the Brain}

The cell membrane consists of three main classes of amphipathic lipids: phospholipids, glycolipids, and sterols (Mouritsen, 2005). The relative amount of each lipid depends upon the type of cell, but in the majority of cases phospholipids are the most abundant. The fatty chains in phospholipids and glycolipids usually contain an even number of carbon atoms, typically between 16 and 20. The 16- and 18-carbon fatty acids are the most common. Fatty acids may be saturated or unsaturated, with the configuration of the double bonds nearly always "cis". The length and the degree of unsaturation of fatty acid chains have a profound effect on membrane fluidity (Gray, Groeschler, Le et al., 2002) as unsaturated lipids create a kink, preventing the fatty acids from packing together as tightly, thus decreasing the melting temperature (increasing the fluidity) of the membrane (Marsh, 2010; Yang, Sheng, Sun et al., 2011). Due to the importance and role of the cell membrane in transport and integrity of cells, these properties affect the functioning of cells including neurons. How this results in behavior of organs such as the brain, composed of individual cells communicating with other cells via membrane-based events, is an important question that needs elucidation.

The space between the phospholipid molecules (polar head) decreases when the fatty acids are saturated and increases when unsaturated (see Figure 1), it determines the molecular refolding caused by the presence of double bonds. After the second double bond, the molecule folds up further reducing the space when compared to linoleic acid (C18:2). As is possible to see in Figure 2, in fact, the alpha-linolenic acid omega 3 (C18:3) occupies a smaller space.

Linoleic acid is the fatty acid that occupies, within the membrane lipid bilayer the greatest space (see Figure 2).

In 1929 Burr and Burr discovered the characteristics of essentiality of two fatty acids: linoleic acid and $\alpha$ linolenic acid (Burr \& Burr, 1929). These acids belong, respectively, to the series of n-6 and n-3 fatty acids; that is to say that they have a final carbon-carbon double bond in the n- 6 and n-3 position, counting from the methyl end.

In nutrition, the concept of essentiality means that a nutrient cannot be synthesized by the organism and its intake by food is required. The absolute lack of the two fatty acids in a person is manifested by severe and progressive pathological phenomena affecting organs and systems, and may lead to death. The two fatty acids have a complex metabolic activity which, through the action of desaturases and elongases, produce, respectively, 


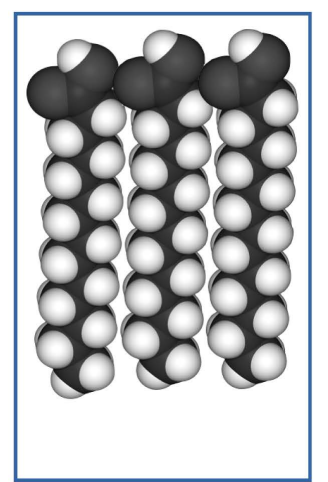

Saturated fatty acids Increased viscosity

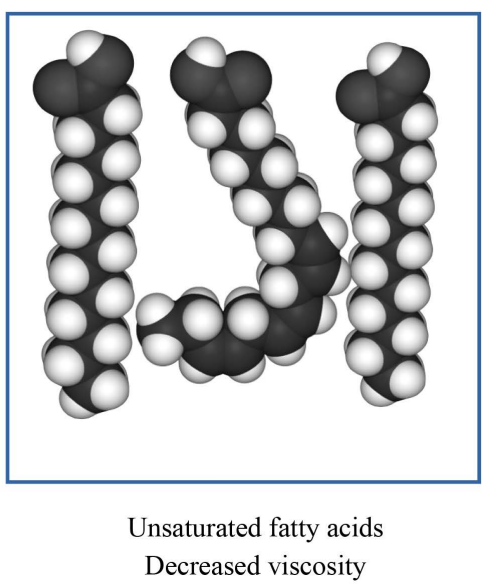

Figure 1. Schematic of the structural differences between saturated and unsaturated fatty acids.

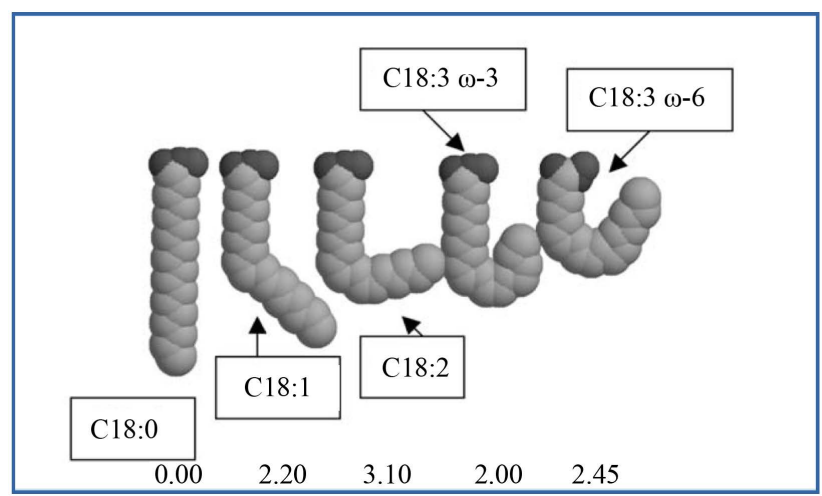

Figure 2. From left to right: Saturated fatty acids, monounsaturated fatty acid, di-unsaturated fatty acid (C18:2 n-6), tri-unsaturated fatty acid (n-6) and tri-unsaturated fatty acid (n-3) in spatial representation of maximum steric hindrance.

arachidonic acid (C20:4, n-6) (linoleic acid) and eicosapentaenoic acid (C20:5, n-3) and docosahexaenoic acid (C22:6, n-3) ( $\alpha$-linolenic acid), which are commonly known as EPA and DHA, without the possibility of interchange between the two metabolic pathways.

Arachidonic acid and DHA are essential for the growth and chemical maturation of brain, during fetal and neonatal development, of all animals, including humans (Hornstra et al., 1995; Uauy et al., 1990; Neuringer et al., 1986; Cocchi \& Noble, 1992; Cocchi, 1993; Noble \& Cocchi, 1990). Linoleic acid and $\alpha$ linolenic acid are distributed in cell membranes of the whole organism, in variable concentrations. This applies mainly to the linoleic acid, while the $\alpha$-linolenic acid is, in all biological districts, present in very low concentrations (Abbott et al., 2012). This suggests the capacity of functional aspects more complex for the linoleic acid (in addition to the production of arachidonic acid), while for the $\alpha$-linolenic acid, the main role seems to be to ensure the production of the correct amount of EPA and DHA.

In hibernating animals, e.g., if there is an insufficient amount of linoleic acid in brown adipose tissue in the pre hibernation period, this would lead to changes in the flow of calcium ions in the cardiomyocyte, which may result in cardiac arrest (Carneheim, Cannon, \& Nedergaard, 1989). The linoleic acid, unlike other districts, as first reported, inexplicably is present in very small concentrations, close to zero, in the brains of animals (Cocchi, Tonello, Gabrielli et al., 2014) and humans, regardless of age, from the embryo-fetal stage to advanced senescence in all phospholipid fractions (Svennerholm, 1968). Because the values of its concentrations are even lower than the analytical error, therefore, it will likely never be possible to accurately evaluate changes in the concentration of linoleic acid in the brain, and even less, to correlate any change to molecular dynamics corre- 
sponding to possible pathological phenomena. In a particular cell, the platelet, which is known to be a mirror of the neuron in psychiatric disorders (Cocchi, Tonello, \& Gabrielli, 2012a; Takahashi, 1976; Stahl, 1977; Pletscher \& Laubscher, 1980; Da Prada, Cesura, Launay et al., 1988; Kim et al., 1982; Musselman, 1996; Camacho \& Dimsdale, 2000; Plein \& Berk, 2001; Bowden, 2001), were found significant fluctuations of linoleic acid in reference to different psychiatric disorders (Major Depression, Bipolar Disorder, Obsessive Compulsive Disorder and Suicide Attempts) and Ischemic Cardiovascular Disease, i.e. very low concentrations in suicide attempts and cardiovascular disease and high concentrations in Obsessive Compulsive Disorder (Benedetti et al., 2012; Benedetti et al., 2014; Cocchi, Gabrielli, \& Tonello, 2013; Cocchi, Tonello, \& Gabrielli, 2012b, 2012c; Cocchi, Tonello, \& Lercker, 2010; Tonello \& Cocchi, 2010; Cocchi \& Tonello, 2010a, 2010b; Cocchi, Tonello, \& Rasenick, 2010) (see Figure 3).

The Map obtained by the SOM, has classified: Normal subjects (N), Obsessive Compulsive Disorder (1), Major Depression (2), Bipolar Disorder (3), Suicide Attempts (4), Psychosis (5), according to the linoleic acid concentration in different cases (see Figures 5-7).

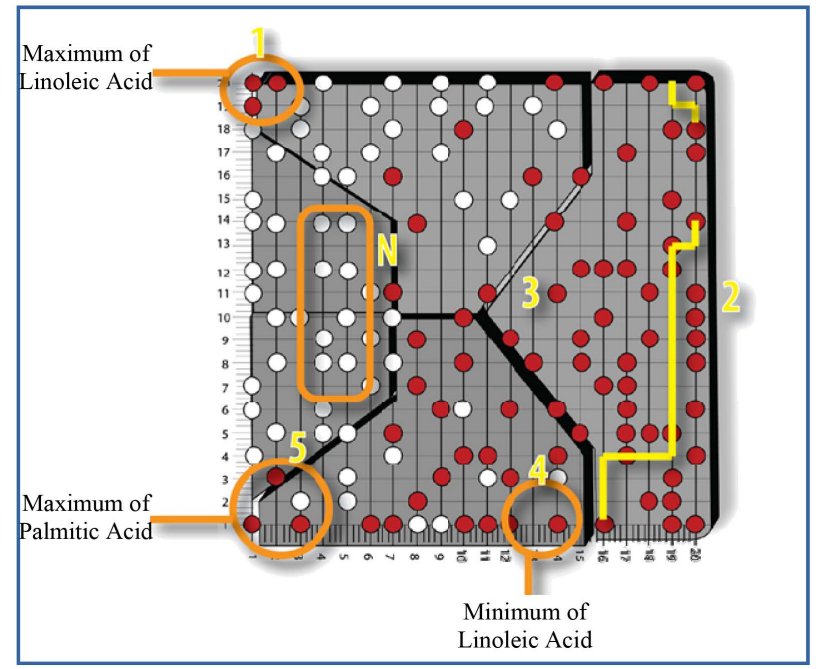

Figure 3. Linoleic acid ranges from the minimum to the maximum and distributes itself, over the Self Organizing Map (SOM) (Stachowska et al., 2004), like a fan (see Figure 4). The self Organizing Map is a type of Artificial Neural Network. It is an important tool to analyze non-linear data. It becomes essential to elaborate in the same time datas with many variables e.g. membrane fatty acids profile. The SOM is an unsupervised competitive-learning network algorithm, which classifies and groups the samples according to the characteristics of similarity. Within the SOM similar items are placed near and different objects are placed far (Kohonen, 2000; Cocchi, Tonello, \& Tsaluchidu et al., 2008).

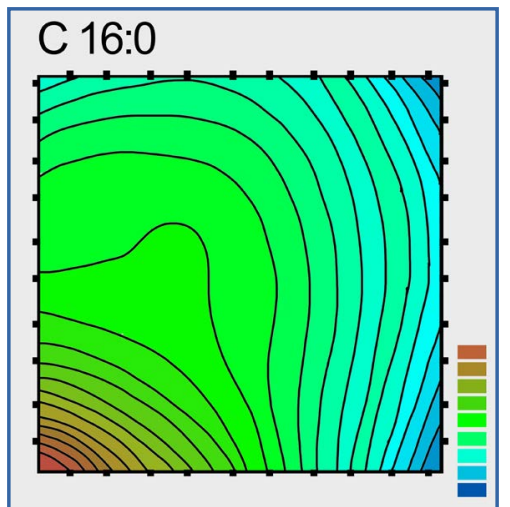

(a)

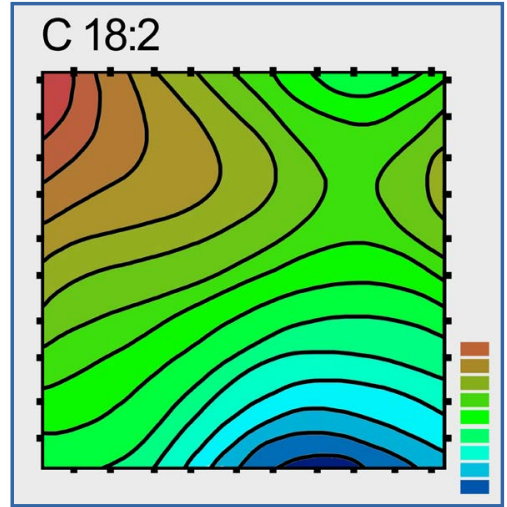

(b)

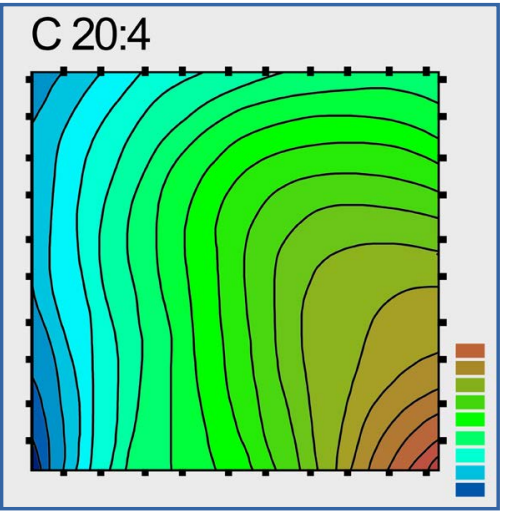

(c)

Figure 4. Curves representing the levels of the fatty acids: (a) palmitic (C16:0); (b) linoleic (C18:2); and (c) arachidonic (C20:4) selected by the SOM, in platelets, for the classification of the Mood Disorders. Brown (maximum concentration) and Blue (minimum concentration). 


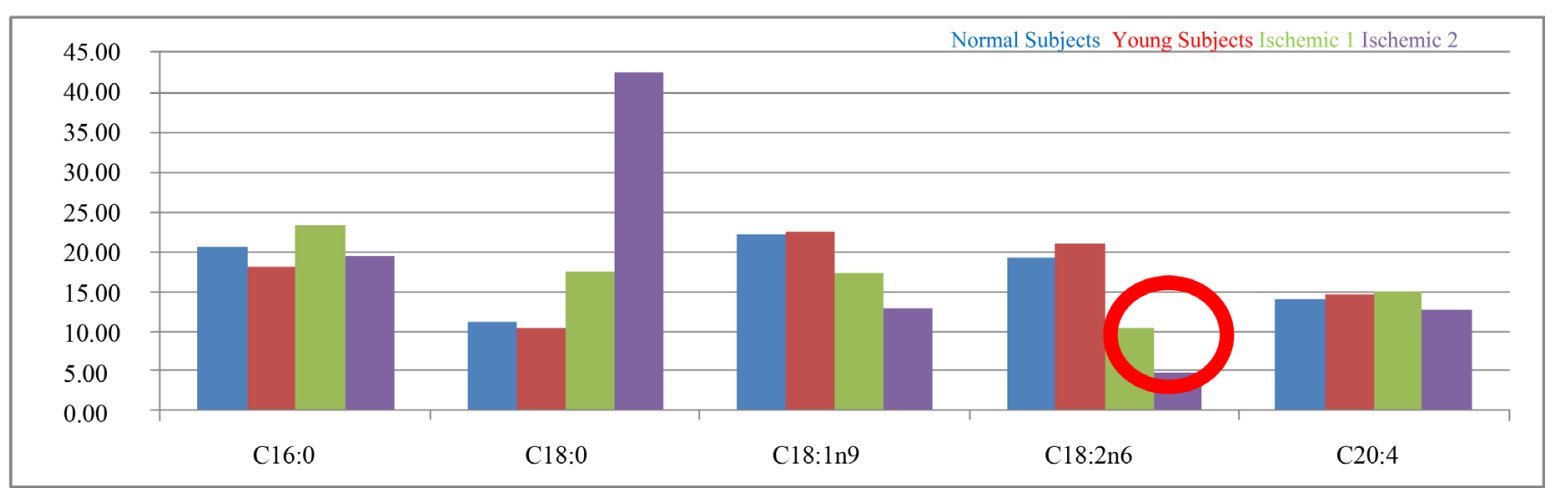

Figure 5. Percent concentration of the platelet's linoleic acid (C18:n-6) in heart ischemic subjects compared to normal (mean age $33.97 \pm 12.4$ ) and young adult (mean age $22.7 \pm 3.7$ ) subjects. Ischemic 1 and 2 are related to two different experiments in subjects who undergo coronarography ( 1 = beginning of the pathology, 2 = advanced and worst pathological conditions). The red circle underlines the lowest concentration of linoleic acid in the ischemic people.

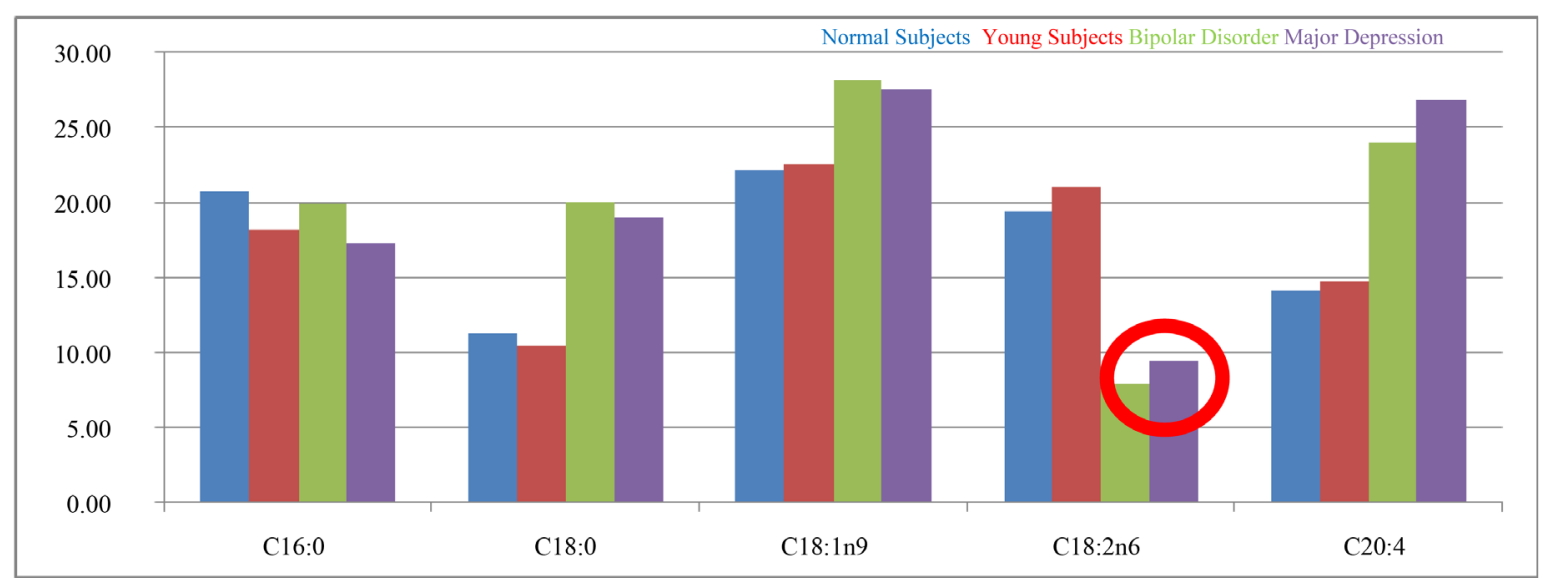

Figure 6. Percent concentration of the platelet's linoleic acid (C18:n-6) in Major Depression and Bipolar Disorder subjects compared to normal and young adult subjects (subjects with Major Depression and Bipolar Disorder have been identified by SOM and confirmed by psychiatric diagnosis, having excluded subjects in first diagnosis). The red circle underlines the lowest concentration of linoleic acid in the ischemic people and depressive subjects.

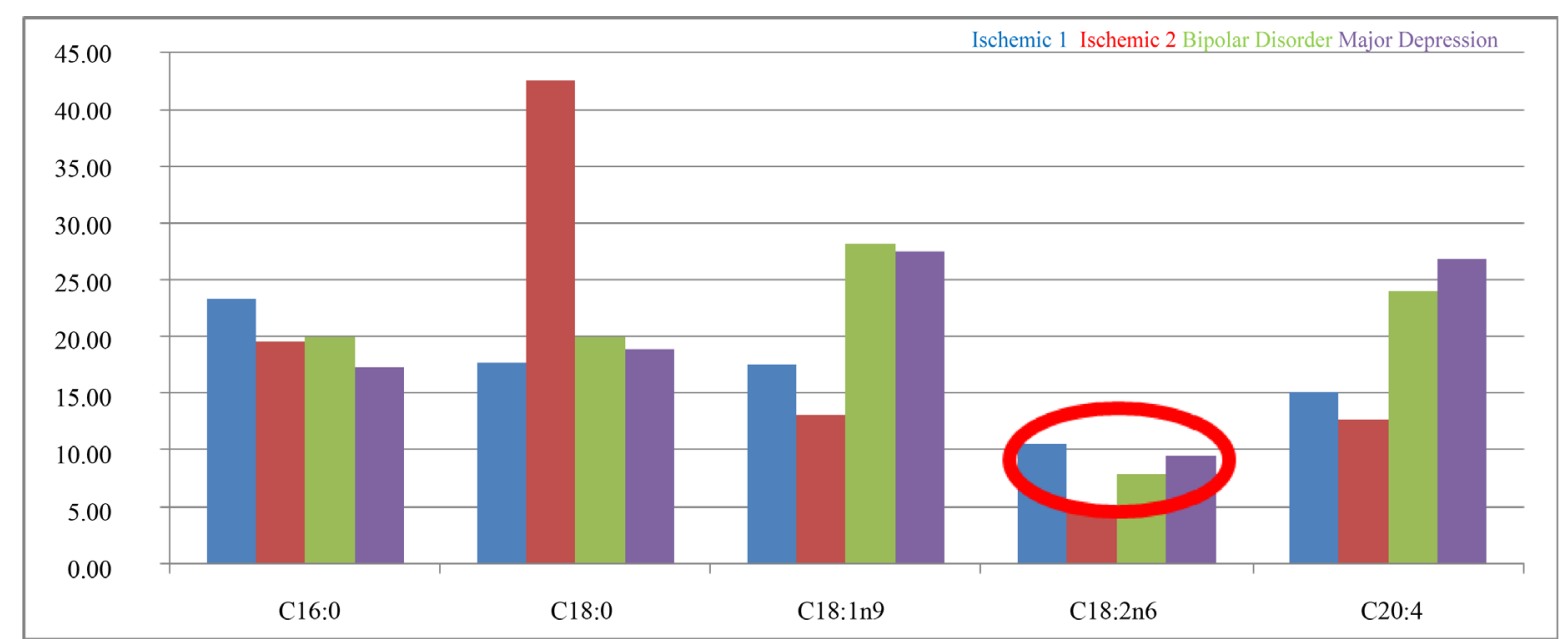

Figure 7. Percent concentration of the platelet's linoleic acid (C18:n-6) in Major Depression and Bipolar Disorder subjects compared to ischemic subjects. The red circle underlines the lowest concentration of linoleic acid in the pathologic subjects studied. 
These figures show clearly that a low linoleic acid concentration is typical of subjects with Coronary Artery Disease and Mood Disorders. It seems that linoleic acid is deeply involved in the molecular properties and functions of the cell membrane in both healthy and pathological cases. Taking into account the characteristic of essentiality of linoleic acid, an absolute zero of linoleic acid concentration in the brain, would, probably, mean the cessation of life. The dramatic effect of the linoleic acid's effects in shown in Figure 8.

In practice, from the biological point of view, the concentration of linoleic acid in the brain appears to be attributable to an unavoidable selectivity of incorporation into the membranes, almost to guarantee the maintenance of the stability of the brain functions. On the other hand, it is known, that the desaturase activity of linoleic acid, in the brain, is negligible (De Mar, 2006) and this further demonstrates that linoleic acid is not subject to metabolic activities of transformation, presumably, to maintain as constant as possible its very low concentration.

We have previously described a striking similarity between platelets and neuron cells in terms of their fatty acid composition in their membranes. As Figure 9(a) shows, there is a marked decrease of the linoleic acid concentration values in the brains of control subjects compared to those involved in suicides (not due to MDMajor Depression) and suicides due to MD (Lalovic et al., 2007). Figure 9(b) shows that the linoleic acid concentration decreases from controls to subjects with Mood Disorders (no suicide cases) and subjects who have attempted a suicide due to psychiatric reasons. Also for very different concentrations the behavior is the same. We have not considered the SD for platelet's fatty acids because the SOM has not used it. In the case of the study in ref. (Lalovic et al., 2007) also if the authors state no significance among the three groups of subjects, the SD of the linoleic acid concentration in the case of suicides for psychiatric reasons is smaller (more than two times) than the others. This means that the value is very stable and that there is a trend to a decrease of Linoleic Acid in suicide for psychiatric reasons.

As of yet, there are no reports on the effects on subjects' behavior as a result of even small variations (both upwards and downwards) in the very low concentration of linoleic acid in the brain.

\section{Linoleic Acid and Symmetry Breaking}

Symmetry breaking in physics describes a phenomenon where even infinitesimally small fluctuations acting on

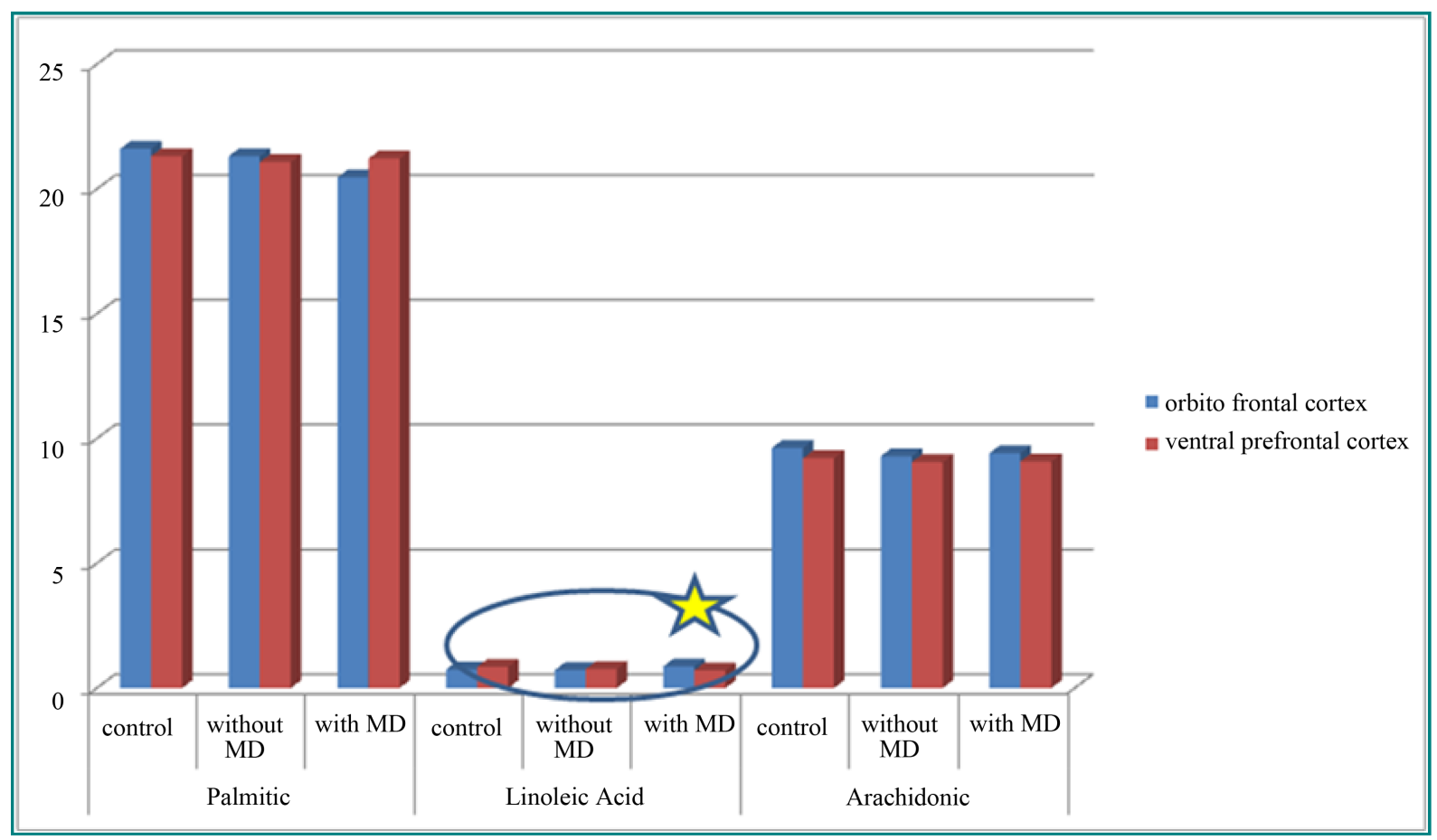

Figure 8. Diagram of two different brain regions showing the very low level of linoleic acid and a different trend in ventral prefrontal cortex in comparison to the orbito frontal cortex (Lalovic et al., 2007). 


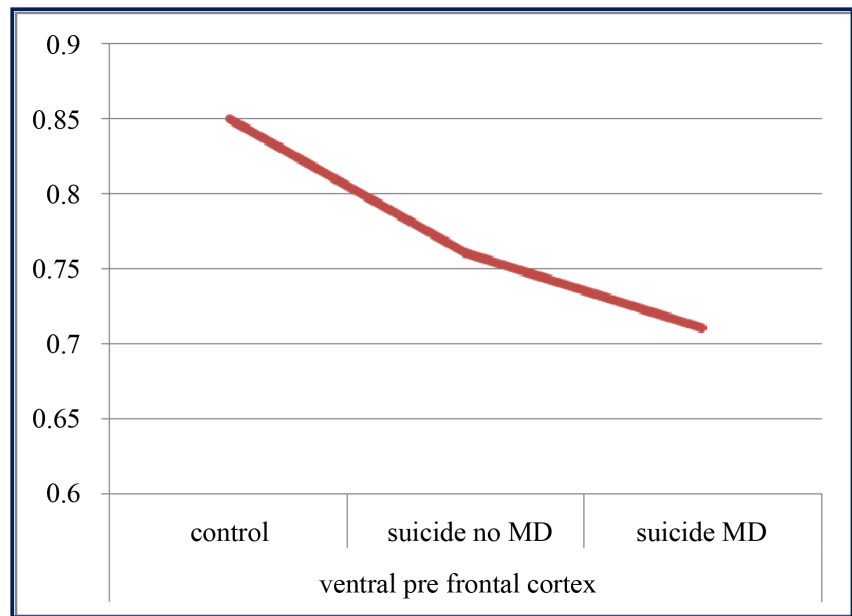

(a)

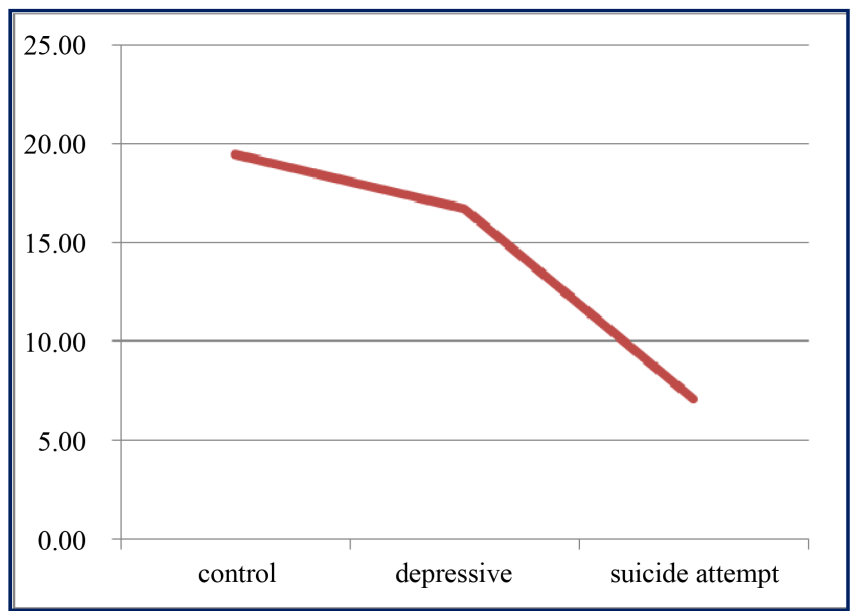

(b)

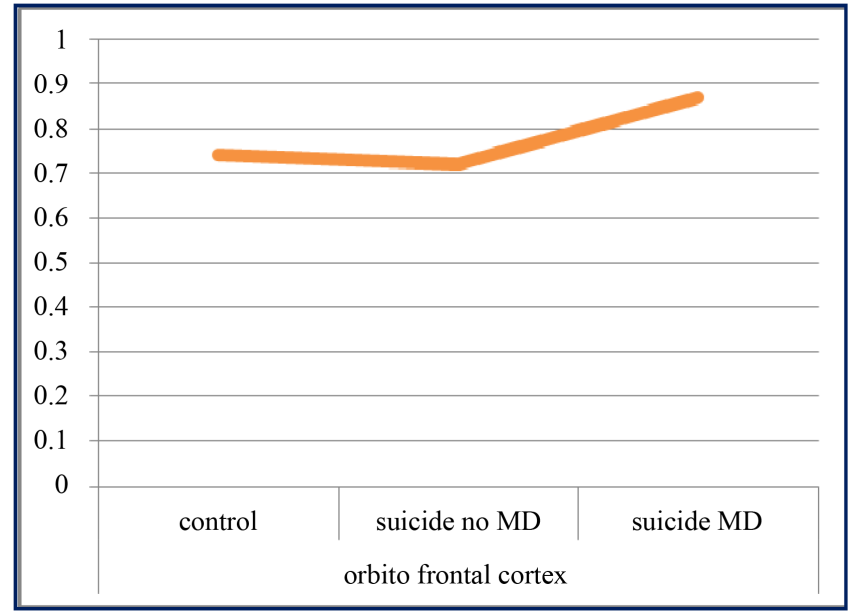

(c)

Figure 9. (a) Percent concentration of linoleic acid in the controls, in suicides not due to MD and suicides due to MD (Lalovic et al., 2007); (b) Percent concentration of linoleic acid in platelets of control, depressed and suicide attempts subjects for psychiatric reasons (Lalovic et al., 2007); (c) The percent concentration of linoleic acid has an opposite trend in the orbito-frontal cortex than in the ventral prefrontal cortex (decision making area), suggesting that this one is critical for suicide ideation (Benedetti et al., 2012). 
a system crossing a critical point decide the system's fate, by determining which branch of a bifurcation curve is taken. This means that the system undergoes instability when it crosses its critical point and instead of a uniquely stable state in the so-called disordered phase, it has more than one (usually two) broken-symmetry (ordered) states. To an outside observer unaware of the fluctuations, the choice of the one of several broken symmetry states may appear arbitrary (Anderson et al., 1997). The measurable quantity that bifurcates is called an order parameter (e.g. magnetization of a ferromagnet) and the variable that is externally varied, which affects the system's behavior is called a control parameter (e.g. the ambient temperature). This process is called symmetry "breaking”, because such transitions usually bring the system from a symmetric but disorderly state (paramagnetism) into one or more ordered states (ferromagnetic domains), which are physically equivalent. In the example of magnetic systems, above the Curie temperature a magnetic system exists in a disordered paramagnetic phase while below the Curie temperature it bifurcates into the ferromagnetic phase characterized by a non-zero net value of magnetization (order parameter). Each orientation of the magnetization vector is equivalent (defining magnetic domains) but this rotational symmetry is broken by an infinitesimal fluctuation of a local magnetic field resulting in the formation of specific magnetic domains with well-defined orientations of their order parameter, i.e. magnetization vectors.

Symmetry breaking phenomena are closely related to phase transitions, many of which (but not all) have underlying broken symmetries. Phase transitions in physical systems have been quantitatively and conceptually elucidated with the advances made by the Renormalization Group Theory in the 1970s. These advances partly derive from the discovery of the role of the control parameters (e.g. temperature and pressure) that drive the systems to instability when approaching their critical values and the resultant changes in the corresponding order parameters (e.g. the value of net magnetization in a ferromagnet) that describe the major physical changes in the critical system under study. The basic underlying principle that is used to determine the equilibrium state of a macroscopic system is the second law of thermodynamics. In the case of thermally and materially isolated systems, this translates into the condition that the entropy $S$ should achieve a maximum value. However, phase transitions usually occur in systems that are not isolated from their surroundings but are in continuous contact with a heat reservoir at a fixed temperature $T$, which requires minimizing the free energy function, $F$.

The most fundamental difference between living systems and the non-living systems in view of thermodynamics is that by definition the former exist in states that are far from thermodynamic equilibrium. Living systems survive only because there is a flux of matter and energy between them and their surroundings, and an export of entropy into their surroundings to compensate for the creation and maintenance of structural order (entropy reduction) and functional organization. Nevertheless, there exist phase transitions in far-from-equilibrium physical systems too, for example the so-called Bénard instability, when a fluid heated from below reaches a critical temperature gradient threshold and makes a transition from a uniform to a convective phase.

In a previous paper on a related topic, Cocchi, M., Gabrielli, F., Pessa, E., Pregnolato, M., Tonello, L., \& Zizzi, P. (2012) introduced the idea of symmetry breaking in mental states with a different approach where it was related to major depression and bipolar disorder through the b2 index, corresponding to two different pathological conditions which involve all the three fatty acids and not only the linoleic acid. In this case the symmetry breaking is related to the linoleic acid but exclusively related to the brain and not to mental pathology, which is a pure biological observation. This is independent of pathology but can explain that the phylogeny has acted only in the complexity of the brain connections and not on the membrane structure. In other words, the hardware is the same for all animals and guarantees the brain stability but the brain's software changes according to the stage of animal evolution unto the man (Cocchi \& Minuto, 2015).

\section{Linoleic Acid: A Critical Element of Psychopathologies?}

In this paper we postulate that there is a critical concentration of just the linoleic acid in the membrane of neuronal cells, which defines a symmetry breaking situation with respect to the human mental health. In other words, the concentration of the linoleic acid in neuronal membranes is a control parameter for the mental state of the subject. Deviations from this critical concentration lead to a broken symmetry of the mental state and either lead to the patient's Bipolar Disorder or Major Depression. These manifestations of pathological mental states can be characterized as the emergence of a non-zero value of the order parameter. In mentally healthy subjects, the concentrations of fatty acids fall within a range of values shown in Figure 3 and Figure 4. The regions on the phase diagram of the fatty acid concentrations that correspond to serious mental state pathologies have been re- 
cently delineated by Cocchi and Tonello and are also shown here in Figures 3-7. Since a transition between the normal mental state and various pathological types appears to be extremely sensitive to the fatty acid concentration ratio, we postulate this is a phase transition in the phase space of mental states and this concentration ratio is a suitable control parameter (a knob) which determines the onset of bifurcation from a disordered state (normal mental state) to any of the broken symmetry states (mental pathologies such as the Bipolar Disorder, Major Depression, etc.). Naturally, if this is an appropriate representation, new and important questions arise, namely: "is it a reversible transition?”, if so, how can the fatty acid concentration ratio be manipulated back to the normal range? Can there be other means of restoring the symmetry of the mental state by the use of external control parameters (e.g. pharmacological agents, electro-stimulation, nutritional means, etc.)?

Finally, it should be noted that there is also a class of systems exhibiting quantum critical phenomena (Sachdev, 2011) and a quantum version of symmetry breaking. In physical systems, they generally are limited to extremely low temperatures but this is dictated by the fact that thermal equilibrium is required. Because living systems, in particular, conscious ones operate far from thermal equilibrium, it is possible that the associated quantum symmetry breaking phenomena may occur at high enough temperatures required by human physiology.

\section{Conclusion}

Two questions arise simultaneously as a result of the above discussion:

1) Could the concentration of linoleic acid in neuronal membranes represent a bifurcation point in a symmetrybreaking phenomenon in the brain with a finely defined critical value?

2) Could linoleic acid conceivably result in the maintenance of quantum effects in the brain?

Answers to these questions cannot come exclusively from biology, but should be the subject to investigations involving quantum physics and physics of critical phenomena. Above, we have elaborated on potential discussion points in regard to these questions from a phenomenological perspective. A mechanistic molecular-level model of these effects is still required to provide deeper insights. Finding a molecular "tipping point" such as the critical concentration of linoleic acid in the membrane of neuronal cells is of major importance to both diagnostics and therapeutics of mental disorders. In this paper we have provided a conceptual framework and some empirical data that support this radically new and important idea.

\section{Acknowledgements}

The authors thank their colleagues Drs. Don Mender, Mark Rasenick, Gordon Globus, Gustav Bernroider and Fabio Gabrielli for numerous insightful discussions on this topic.

\section{References}

Abbott, S. K., Elsea, P. L., Atkins, T. A., \& Hulberta, A. J. (2012). Fatty Acid Composition of Membrane Bilayers: Importance of Diet Polyunsaturated Fat Balance. Biochimica et Biophysica Acta (BBA)—Biomembranes, 1818, 1309-1317. http://dx.doi.org/10.1016/j.bbamem.2012.01.011

Abeles, M. (1991). Corticonics: Neural Circuits of the Cerebral Cortex. Cambridge: Cambridge University Press. http://dx.doi.org/10.1017/CBO9780511574566

Anderson, P. W. (1997). Basic Notions of Condensed Matter Physics. Reading: Addison-Wesley.

Benedetti, S., Bucciarelli, S., Canestrari, F., Catalani, S., Colomba, M. S., Gregorini, A., Mandolini, S., Marconi, V., Mastrogiacomo, A. R., Rasenick, M., Silvestri, R., Tagliamonte, M. C., Tonello, L., Venanzini, R., \& Cocchi, M. (2012). Molecular Changes in Mood Disorders Results of the Marche Region Special Project. NeuroQuantology, 10, S1-S28.

Benedetti, S., Bucciarelli, S., Canestrari, F., Catalani, S., Mandolini, S., Marconi, V., Mastrogiacomo, A., Silvestri, R., Tagliamonte, M., Venanzini, R., Caramia, G., Gabrielli, F., Tonello, L., \& Cocchi, M. (2014). Platelet’s Fatty Acids and Differential Diagnosis of Major Depression and Bipolar Disorder through the Use of an Unsupervised Competitive-Learning Network Algorithm (SOM). Open Journal of Depression, 3, 52-73. http://dx.doi.org/10.4236/ojd.2014.32011

Bowden, C. L. (2001). Strategies to Reduce Misdiagnosis of Bipolar Depression. Psychiatric Services, 52, 51-55. http://dx.doi.org/10.1176/appi.ps.52.1.51

Brown, J. A., \& Tuszynski, J. A. (1997). Dipole Interactions in Axonal Microtubules as a Mechanism of Signal Propagation. Physical Review E, 56, 5834-5840. http://dx.doi.org/10.1103/PhysRevE.56.5834

Burr, G. O., \& Burr, M. M. (1929). A New Deficiency Disease Produced by the Rigid Exclusion of Fat from the Diet. Journal of Biological Chemistry, 82, 345-367. 
Camacho, A., \& Dimsdale, J. E. (2000). Platelets and Psychiatry: Lessons Learned from Old and New Studies. Psychosomatic Medicine, 62, 326-336. http://dx.doi.org/10.1097/00006842-200005000-00006

Carneheim, C., Cannon, B., \& Nedergaard, J. (1989). Rare Fatty Acids in Brown Fat Are Substrates for Thermogenesis during Arousal from Hibernation. The Wenner-Gren Institute, University of Stockholm, S-106 91 Stockholm, Sweden.

Cocchi, M. (1993). The Significance of n-3 Fatty Acid in Fetal/Neonatal Development and Some Alternative Sources. Proceedings of the Nutrition Society, 52, 224A.

Cocchi, M., \& Minuto, C. (2015). Linoleic Acid: A Milestone in Brain Evolution? Humane Evolution, 30, 245-257.

Cocchi, M., \& Noble, R. C. (1992). Il ruolo del DHA nello sviluppo embrionale. Nutrizione, 5, 23-26.

Cocchi, M., \& Tonello, L. (2010a). Running the Hypothesis of a Bio Molecular Approach to Psychiatric Disorder Characterization and Fatty Acids Therapeutical Choices. Annals of General Psychiatry, 9, S26.

http://dx.doi.org/10.1186/1744-859X-9-S1-S26

Cocchi, M., \& Tonello, L. (2010b). Bio Molecular Considerations in Major Depression and Ischemic Cardiovascular Disease. Central Nervous System Agents in Medicinal Chemistry, 10, 97-107. http://dx.doi.org/10.2174/187152410791196378

Cocchi, M., Gabrielli, F., \& Tonello, L. (2013). Platelet’s Fatty Acids Secrets in Coronary Artery Disease (CAD). Letter to the Editor, BMJ, 28 October 2013.

Cocchi, M., Gabrielli, F., Pessa, E., Pregnolato, M., Tonello, L., \& Zizzi, P. (2012). Major Depression and Bipolar Disorder: The Concept of Symmetry Breaking. NeuroQuantology, 10, 676-687. http://dx.doi.org/10.14704/nq.2012.10.4.577

Cocchi, M., Tonello, L., \& Gabrielli, F. (2012a). Considerations on Blood Platelets: A Neuron’s Mirror for Mood Disorders? Open Journal of Blood Diseases, 2, 22-29. http://dx.doi.org/10.4236/ojbd.2012.22005

Cocchi, M., Tonello, L., \& Gabrielli, F. (2012b). Possible Roles of Cell Membrane \& Cytoskeleton in Quantum Aspect of Psychiatry. Journal of Consciousness Exploration \& Research, 3, 1082-1100.

Cocchi, M., Tonello, L., \& Gabrielli, F. (2012c). Molecular Uniqueness of Major Depression: Biological Remarks and Theoretical Implications. Journal of Consciousness Exploration \& Research, 3, 380-391.

Cocchi, M., Tonello, L., \& Lercker, G. (2010). Fatty Acids, Membrane Viscosity, Serotonin and Ischemic Heart Disease. Lipids in Health and Disease, 9, 97. http://dx.doi.org/10.1186/1476-511X-9-97

Cocchi, M., Tonello, L., \& Rasenick, M. M. (2010). Human Depression: A New Approach in Quantitative Psychiatry. Annals of General Psychiatry, 9, 25. http://dx.doi.org/10.1186/1744-859X-9-25

Cocchi, M., Tonello, L., Gabrielli, F., \& Minuto, C. (2014). Human and Animal Brain Phospholipids Fatty Acids, Evolution and Mood Disorders. Journal of Phylogenetics \& Evolutionary Biology, 2, 128.

Cocchi, M., Tonello, L., Tsaluchidu, S., \& Puri, B. K. (2008). The Use of Artificial Neural Networks to Study Fatty Acids in Neuropsychiatric Disorders. BMC Psychiatry, 8, S3. http://dx.doi.org/10.1186/1471-244X-8-S1-S3

Craddock, T. J. A., Hameroff, S. R., \& Tuszynski, J. A. (2012). Cytoskeletal Signaling: Is Molecular Memory Encoded in Microtubule Lattices by CaMKII Phosphorylation. PLoS Computational Biology, 8, e1002421.

http://dx.doi.org/10.1371/journal.pcbi.1002421

Craddock, T. J. A., Priel, A., \& Tuszynski, J. A. (2014). Keeping Time: Could Quantum Beating in Microtubules Be the Basis for the Neural Synchrony Related to Consciousness? Journal of Integrative Neuroscience, 13, 293-311. http://dx.doi.org/10.1142/S0219635214400019

Craddock, T. J. A., St. George, M., Freedman, H., Barakat, K. H., Damaraju, S., \& Tuszynski, J. A. (2012). Computational Predictions of Volatile Anesthetic Interactions with the Microtubule Cytoskeleton: Implications for Side Effects of General Anesthesia. PLoS ONE, 7, e37251. http://dx.doi.org/10.1371/journal.pone.0037251

Da Prada, M., Cesura, A. M., Launay, J. M., \& Richards, J. G. (1988). Platelets as a Model for Neurones? Cellular and Molecular Life Sciences, 44, 115-126. http://dx.doi.org/10.1007/BF01952193

De Mar, J. C. (2006). Brain Elongation of Linoleic Acid Is a Negligible Source of Arachidonic Acid in the Brain Phospholipid of Adult Rats. Biochimica et Biophysica Acta, 1761, 1050-1059. http://dx.doi.org/10.1016/j.bbalip.2006.06.006

Donati, R. J., \& Rasenick, M. M. (2003). G Protein Signaling and the Molecular Basis of Antidepressant Action. Life Sciences, 73, 1-17. http://dx.doi.org/10.1016/S0024-3205(03)00249-2

Friesen, D. E., Craddock, T. J. A., Karla, A., \& Tuszynski, J. A. (2015). Biological Wires, Communication Systems and Implications for Disease. Biosystems, 127, 14-27. http://dx.doi.org/10.1016/j.biosystems.2014.10.006

Gray, J., Groeschler, S., Le, T., \& Gonzalez, Z. (2002). Membrane Structure (SWF). Davidson, NC: Davidson College.

Hameroff, S., Nip, A., Porter, M., \& Tuszynski, J. A. (2002). Conduction Pathways in microtubules. Biological quantum Computation, and Consciousness. Biosystems, 64, 149-168. http://dx.doi.org/10.1016/S0303-2647(01)00183-6

Hornstra, G., Al, M. D. M., van Houwelingen, A. C., \& Foreman-van Drongelen, M. M. H. P. (1995). Essential Fatty Acid in Pregnancy and Early Humane Development. European Journal of Obstetrics \& Gynecology and Reproductive Biology, 61, 
57-62. http://dx.doi.org/10.1016/0028-2243(95)02153-J

Kim, H. L., Plaisant, O., Leboyer, M., Gay, C., Kamal, L., Devynck, M. A., \& Meyer, P. (1982). Reduction of Platelet Serotonin in Major Depression (Endogenous Depression). Comptes Rendus des Séances de l'Académie des Sciences, Série III, 295, 619-622.

Kohonen, T. (2000). Self Organizing Maps (3rd ed.). Berlin: Springer-Verlag.

Lalovic, A., Levy, E., Canetti, L., Sequeira, A., Montoudis, A., \& Turecki, G. (2007). Fatty Acid Composition in Post-Mortem Brains of People Who Completed Suicide. Journal of Psychiatry \& Neuroscience, 32, 363-370.

Marsh, D. (2010). Structural and Thermodynamic Determinants of Chain-Melting Transition Temperatures for Phospholipid and Glycolipids Membranes. Biochimica et Biophysica Acta, 1798, 40-51.

http://dx.doi.org/10.1016/j.bbamem.2009.10.010

Mouritsen, O. (2005). Life-As a Matter of Fat: The Emerging Science of Lipidomics. Berlin: Springer.

Musselman, D. L., Tomer, A., Manatunga, A. K., Knight, B. T., Porter, M. R., Kasey, S., Marzec, U., Harker, L. A., \& Nemeroff, C. B. (1996). Exaggerated Platelet Reactivity in Major Depression. American Journal of Psychiatry, 153, 13131317. http://dx.doi.org/10.1176/ajp.153.10.1313

Neuringer, M., Connors, W. E., Lin, D. S., Barstard, L., \& Luck, S. (1986). Biochemical and Functional Effects of Prenatal and Postnatal Omega 3 Fatty Acid Deficiency on Retina and Brain in Rhesus Monkeys. Proceedings of the National Academy of Sciences of the United States of America, 83, 4021-4025. http://dx.doi.org/10.1073/pnas.83.11.4021

Nicholls, J. G., Martin, A. R., Fuchs, P. A. Brown, D. A., Diamond, M. E., \& Weisblat, D. (2011). From Neuron to Brain (5th ed.). New York: Sinauer Associates.

Noble, R. C., \& Cocchi, M. (1990). Lipid Metabolism and the Neonatal Chicken. Progress in Lipid Research, 29, $107-140$. http://dx.doi.org/10.1016/0163-7827(90)90014-C

Plein, H., \& Berk, M. (2001). The Platelet as a Peripheral Marker in Psychiatric Illness. Human Psychopharmacology, 16, 229-236. http://dx.doi.org/10.1002/hup.251

Pletscher, A., \& Laubscher, A. (1980). Blood Platelets as Models for Neurons: Uses and Limitations. Journal of Neural Transmission Supplementa, 16, 7-16. http://dx.doi.org/10.1007/978-3-7091-8582-7_2

Rasenick, M. M., Donati, R. J., Popova, J. S., \& Yu, J. Z. (2004). Tubulin as a Regulator of G-Protein Signaling. Methods in Enzymology, 390, 389-403. http://dx.doi.org/10.1016/S0076-6879(04)90024-9

Sachdev, S. (2011). Quantum Phase Transitions (2nd ed.). Cambridge: Cambridge University Press. http://dx.doi.org/10.1017/CBO9780511973765

Stachowska, E., Gutowska, I., Dołegowska, B., Chlubek, D., Bober, J., Rać, M., Gutowski, P., Szumilowicz, H., \& Turowski, R. (2004). Exchange of Unsaturated Fatty Acids between Adipose Tissue and Atherosclerotic Plaque Studied with Artificial Neural Networks. Prostaglandins, Leukotrienes and Essential Fatty Acids, 70, 59-66. http://dx.doi.org/10.1016/j.plefa.2003.08.021

Stahl, S. M. (1977). The Human Platelet. A Diagnostic and Research Tool for the Study of Biogenic Amines in Psychiatric and Neurologic Disorders. Archives of General Psychiatry, 34, 509-516. http://dx.doi.org/10.1001/archpsyc.1977.01770170019001

Svennerholm, L. (1968). Distribution and Fatty Acid Composition of Phosphoglycerides in Normal Human Brain. Journal of Lipid Research, 9, 570-579.

Takahashi, S. (1976). Reduction of Blood Platelet Serotonin Levels in Manic and Depressed Patients. Folia Psychiatrica et Neurologica Japonica, 30, 476-486. http://dx.doi.org/10.1111/j.1440-1819.1976.tb02670.x

Tonello, L., \& Cocchi, M. (2010). The Cell Membrane: Is It a Bridge from Psychiatry to Quantum Consciousness? NeuroQuantology, 8, 54-60. http://dx.doi.org/10.14704/nq.2010.8.1.268

Uauy, R. D., Birch, D. G., Birch, E. E., Tyson, J. E., \& Hoffman, D. R. (1990). Effect of Dietary Omega-3 Fatty Acids on Retinal Function of Very-Low-Birth-Weight Neonates. Pediatric Research, 28, 485-492. http://dx.doi.org/10.1203/00006450-199011000-00014

Woolf, N. J., Priel, A., \& Tuszynski, J. A. (2010a). Nanoneuroscience: Structural and Functional Roles of the Neuronal Cytoskeleton in Health and Disease. Heidelberg: Springer Verlag. http://dx.doi.org/10.1007/978-3-642-03584-5

Woolf, N. J., Priel, A., \& Tuszynski, J. A. (2010b). Neural Cytoskeleton Correlates of Learning and Memory. Journal of Biological Physics, 36, 3-21. http://dx.doi.org/10.1007/s10867-009-9153-0

Yang, X. G., Sheng, W. W,, Sun, G. Y., \& Lee, J. C. M. (2011). Effects of Fatty Acid Unsaturation Numbers on Membrane Fluidity and $\alpha$-Secretase-Dependent Amyloid Precursor Protein Processing. Neurochemistry International, 58, 321-329.

http://dx.doi.org/10.1016/j.neuint.2010.12.004 